\title{
COMPORTAMENTO MOTOR E COGNIÇÃO EM CRIANÇAS PARTICIPANTES E NÃO PARTICIPANTES DE UM PROGRAMA DE MOTRICIDADE
}

\section{ARTIGO ORIGINAL}

PRADO, Rosa Luciana ${ }^{1}$

MENDONÇA, Fernando Cruz de ${ }^{2}$

SILVA, Jadisson Gois da ${ }^{3}$

PRADO, Rosa Luciana. MENDONÇA, Fernando Cruz de. SILVA, Jadisson Gois da. Comportamento motor e cognição em crianças participantes e não participantes de um programa de motricidade. Revista Científica Multidisciplinar Núcleo do Conhecimento. Ano 05, Ed. 08, Vol. 03, pp. 168-180. Agosto de 2020. ISSN: 24480959, Link de acesso: https://www.nucleodoconhecimento.com.br/educacaofisica/comportamento-motor

\section{RESUMO}

Introdução: A identificação de níveis de desenvolvimento e funcionalidade do indivíduo é primordial para o desenvolvimento de programas interventivos que tenham como propósito potencializar progresso de novas competências, remediar dificuldades já

\footnotetext{
${ }^{1}$ Mestrado Saúde e Ambiente pela Universidade Tiradentes (UNIT); Especialização em Fisiologia do exercício (Centro Universitário Estácio de Sergipe); Graduação em Educação Física (universidade Federal de Sergipe).

2 Graduação em Educação Física Bacharelado (Centro Universitário Estácio de Sergipe).

${ }^{3}$ Especialização residente multiprofissional em Saúde mental (Hospital Universitário da Universidade Federal de Sergipe); Graduação em Educação Física (Centro Universitário Estácio de Sergipe).
} 
determinadas e/ou desenvolver novos métodos de ensino. Objetivo: Verificar a efetividade de um programa de motricidade para crianças (3-5 anos), identificando diferenças significativas entre grupos participantes (G1) e não-participantes (G2) de um programa de motricidade de uma escola da rede particular da cidade de Aracaju, Sergipe, Brasil. Metodologia: Utilizou-se pesquisa de campo experimental com abordagem transversal. Participaram do estudo 46 crianças (3-5 anos) de ambos os gêneros sendo 23 participantes de um programa interventivo de motricidade (G1) e 23 não-participantes (G2). A amostra foi selecionada por conveniência tendo em vista critérios estabelecidos para inclusão dos sujeitos: Idade, matrícula, participar do programa (G1) e não participar do programa (G2), ter assinado Termo de Assentimento. Para avaliação da Idade Motora (IM) foi utilizado o Kit da Escala de Desenvolvimento Motor (EDM) (ROSA NETO, 2014) e para identificação da conservação cognitiva a bateria Piagetiana (PIAGET, 1998). Resultados: Evidenciouse homogeneidade na distribuição dos dados. A média da idade motora apresentou diferenças significativas entre os grupos ( $G 1=75,04 \pm 4,7 ; G 2=38,61 \pm 3,7)(t=6.063$; $p=0,00)$; na avaliação Piagetiana de Cognição foi percebido mais sujeitos $(69,5 \%)$ concentrados nos níveis II e III significando melhores ajustes cognitivos em detrimento da faixa etária enquanto o G2 prevaleceu concentração no nível I (73,9\%); Houve diferenças significativas entre os grupos (G1; G2) tanto na Idade Motora Geral quanto nos níveis de cognição $(p \leq 0,05)$. A partir dos achados obtidos na presente pesquisa constatou-se mudanças positivas confiáveis nos elementos das motricidades que passaram por estimulação. Conclusão: Diante a análise, percebe-se mudanças confiáveis quando as áreas psicomotoras são estimuladas em programas revelando que crianças inseridas nesse contexto apresentam melhoras nas áreas estimuladas, portanto, crianças que praticam programa de motricidade tem mais probabilidade de melhorar seu desenvolvimento cognitivo do que crianças não praticantes.

Palavras Chave: Motricidade, crianças, cognição.

\section{INTRODUÇÃO}

Nos primeiros anos de vida, o desenvolvimento motor caracteriza-se como a conquista de amplas habilidades motoras, que por meio do domínio corporal permite ao 
indivíduo realizar competências básicas, como locomover-se e manipular inúmeros objetos diferentes. Algumas dessas habilidades são solicitadas já no começo da vida, sendo fundamental para ser utilizada no cotidiano da criança, tanto nas suas tarefas domiciliares quanto escolares (SANTOS; DANTAS; OLIVEIRA, 2004).

Desse modo, a evolução motora caracteriza-se como uma modificação constante do indivíduo ao longo da vida, sendo que os fatores biológicos e circunstâncias do ambiente em que a criança se encontra determinam as variações no desempenho motor, assim como o influenciam, modificando-o (HAYWOOD; GETCHELL, 2004).

Para tal, o diagnóstico do desenvolvimento motor possibilita aos pesquisadores reconhecerem as causas que tornam o movimento limitado, proporcionando a tomada de decisões sobre as capacidades e/ou parâmetros motores que devem ser focalizados nos programas, no período de ação para cada habilidade e, nas metas de atuação da criança. Esse método contínuo de mudanças poder ser observado nas fases da vida, a partir do qual tem sua ampliação diretamente associada à idade (HAYWOOD; GETCHELL, 2004).

Sendo assim, cabe destacar que existem vários motivos que conduzem ao interesse gradativo pelo desenvolvimento motor, entre eles estão as reações do diagnóstico do crescimento e evolução do indivíduo, reabilitação da criança com atrasos ou desvios de desenvolvimento e adaptações de ambientes e funções motoras nos estágios de desenvolvimento. Essas alterações motoras qualitativas e quantitativas são motivos de constantes pesquisas, não possui somente o intuito de descrever modificações do comportamento motor, mas também possibilita investigações que possam prognosticar essas variáveis (SANTOS; DANTAS; OLIVEIRA, 2004).

Desta forma, a identificação de níveis de desenvolvimento e funcionalidade do indivíduo torna-se fundamental para elaboração de programas interventivos que tenham como objetividade potencializar o progresso de novas competências, remediar dificuldades já determinadas e/ou desenvolver novos métodos de movimentos (CAMPOS et al., 2008). 
Nesse sentido, o presente estudo objetivou-se verificar e discutir a efetividade de um programa de motricidade para crianças 3-5 anos de uma escola da rede particular de ensino no município de Aracaju, Sergipe.

\section{MATERIAIS E MÉTODOS}

A pesquisa foi caracterizada como experimental e transversal por meio da investigação comparativa de dois grupos de crianças (HULLEY; NEWMAN; CUMMINGS, 2003; SILVA, 2004). O primeiro grupo participante de um programa de motricidade (grupo experimental - G1) e o segundo com crianças que não participam de atividade motoras formais (grupo controle - G2). Fizeram parte desse estudo 46 crianças (28 meninos e 18 meninas), com idade de 3-5 anos e matriculadas em uma escola da rede privada da cidade de Aracaju, Sergipe.

Todas as crianças foram selecionadas por conveniência, identificadas a partir da sua participação no programa de motricidade $(\mathrm{G} 1)$ e as demais crianças por sorteio aleatório, tendo em vista critérios de inclusão: 1) O aluno precisa estar matriculado, apresentar frequência regular nas atividades; 2) apresentar termo de assentimento livre e esclarecido (TALE) assinado pelos pais ou responsável; 3) a criança precisa ter interesse de participar da coleta de dados; 4) não possuir algum diagnóstico clínico, complexidade de aprendizagem, mudanças comportamentais ou mentais, déficits sensoriais, deficiências físicas que afete o desenvolvimento motor ou cognitivo; 5) ambos os sexos, com idade entre 3 a 5 anos.

Para avaliação da Idade Motora (IM), foi utilizada a Escala de Desenvolvimento Motor (EDM), da qual demonstrou a (IM), o quociente motor geral (QMG), e os quocientes das regiões motoras específicas como: Motricidade fina (óculo manual), motricidade global (coordenação), equilíbrio (postura estática), esquema corporal (imitação de postura, rapidez), organização espacial (percepção do espaço), organização temporal (linguagem, estruturas temporais) e lateralidade (mãos, olhos e pés) (ROSA NETO, 2014). 
Para avaliação da competência intelectual, foi aplicado o teste Piagetiano - que avalia o desenvolvimento cognitivo (PIAGET, 1998), detecção do nível de pensamento alcançado por crianças com faixa etária de 2-7 anos (ROSA NETO, 2014).

Após a autorização do projeto pelo comitê de Ética em pesquisa ํo 3.294 .819 permissão da instituição para coleta de dados, e a assinatura do (TALE) pelos responsáveis dos participantes do estudo, as crianças foram analisadas e observadas individualmente nos testes EDM e provas Piagetiana, na própria instituição, em local apropriado, com boa iluminação e sem interferência externa, em duas sessões com duração de 30 a 50 minutos cada.

Para análise dos dados foi utilizado o pacote da Microsoft Excel para tabulação dos resultados e organização das tabelas e gráficos e o Pacote estatístico do IBM SPSS Statistics 25 para comparação dos grupos por meio do teste $\mathrm{T}$ de Student e a correlação linear de Pearson para verificação das relações existentes entre as variáveis estudadas (IMG e Cognição).

\section{RESULTADOS}

Os resultados encontrados apontam homogeneidade na distribuição dos dados e não havendo assim distinção entre os gêneros estudados quanto as variáveis da Idade Motora Geral (IMG) $(t=-0,617 ; p=0,541)$ e da média dos testes Piagetiano para avaliação da cognição $(t=-0,965 ; p=0,34)$.

A tabela 1 aponta as características da amostra $(n=46)$ distribuídos em dois grupos de 23 sujeitos (ambos os gêneros) e os dados estão expressos em (Média + desvio padrão) e de distribuição (frequência) para a variável Cognição (Nível I, II e III). No que concerne a análise da distribuição dos sujeitos nos grupos $(\mathrm{G} 1 ; \mathrm{G} 2)$ as médias foram: IM $(\mathrm{G} 1=75,0)$ e $(\mathrm{G} 2=38,6)$.

Levando-se em consideração os referidos achados é perceptível que o grupo que participa do programa de motricidade (G1) apresentou média significativamente superior ao grupo não participante (G2). Assim, destaca-se que a análise por teste t 
para amostras independentes indica haver diferenças significativas tanto na variável IMG quanto na média do teste de cognição (Piaget).

A distribuição dos sujeitos, por níveis de cognição, está exposta na (tabela 1) e aponta uma concentração dos sujeitos do G1 em nível acima do normal - nível II (60,9\%) enquanto a concentração dos sujeitos do G2 está no nível I (considerado normal para a faixa etária).

Tabela 1. Características da amostra

\begin{tabular}{|l|l|l|}
\hline VARIÁVEIS/GRUPOS & G1 & G2 \\
\hline Amostra $(\mathrm{n})$ & 23 & 23 \\
\hline Idade Cronológica - IC (meses) & $54,91 \pm 2,25$ & $52,43 \pm 2,23$ \\
\hline Idade Motora geral - IMG (meses) & $75,04 \pm 4,7^{\star}$ & $38,61 \pm 3,7^{\star}$ \\
\hline Nível I de cognição & $7(30,4 \%)$ & $17(73,9 \%)$ \\
\hline Nível II de cognição & $14(60,9 \%)$ & $6(26,1 \%)$ \\
\hline Nível III de cognição & $2(8,6 \%)$ & - \\
\hline Média piagetiana & $1,76 \pm 0,53^{\star \star}$ & $1,28 \pm 0,42^{\star *}$ \\
\hline
\end{tabular}

Níveis I, II e III foram classificados de acordo com a média dos resultados das provas ( p 1, p 2, p 3, p 4, p 5, p 6) Nível I (1 - 1,49); Nível II (1,5 - 2,49); Nível III (2,5 - 3). ${ }^{*}(t=6.063 ; p=0,00){ }^{* *}(t=0.813 ; p=0,000)$

Sobre a idade motora geral (IMG) foi possível também realizar uma análise por categoria de classificação como demonstrado na tabela 2 .

Tabela 2. Distribuição quanto a classificação da motricidade de crianças de 3-5 anos participantes e não participantes de um programa

\begin{tabular}{|l|l|l|}
\hline Classificação do QMG & Quantidade de sujeitos & \\
\hline Muito superior & G1 & G2 \\
\hline Superior & 12 & 0 \\
\hline
\end{tabular}




\begin{tabular}{|l|l|l|}
\hline Normal alto & 5 & 2 \\
\hline Normal médio & 3 & 7 \\
\hline Normal baixo & 0 & 1 \\
\hline Inferior & 0 & 3 \\
\hline Muito inferior & 0 & 10 \\
\hline
\end{tabular}

$\mathrm{QMG}=(\mathrm{IMG} / \mathrm{IC}) \times 100$

É possível identificar a distribuição dos sujeitos quanto a classificação da IMG através de indicadores de riscos do Quociente Motor Geral (QMC). Percebe-se que nenhum dos sujeitos participantes do programa encontra-se em situação de riscos, enquanto $69,3 \%$ das crianças não participantes de programa de motricidade encontram-se abaixo da média esperada para sua idade cronológica (tabela 2). O comprometimento da estruturação motora afeta a cognição das crianças ainda em idade de formação.

Enquanto isso, as analises piagetianas expressaram uma interação significativa entre os grupos observados, levando em consideração os escores cognitivos. Não houve uma diferença estatisticamente significativa entre os sexos frente a média dos testes Piagetiano para avaliação da cognição $(t=-0,965 ; p=0,34)$ conforme apresentado na tabela 1. No fator grupo $(\mathrm{G} 1, \mathrm{G} 2)$, houve uma diferença significativa entre os grupos. O G1 que participa do programa de motricidade apresentou evolução positiva e significativa como exploração e manipulação, relacionamento de objetos. Os resultados observados sugerem efeitos positivos da intervenção no desenvolvimento cognitivo de crianças no programa de motricidade.

Tabela 3. Distribuição dos sujeitos de acordo com o nível alcançado nas provas Piagetianas.

\begin{tabular}{|l|l|l|l|l|l|l|l|}
\hline GRUPOS & Nível & Prova & prova & prova & prova & Prova & Prova \\
& alcançado & 1 & 2 & 3 & 4 & 5 & 6 \\
\hline G1* & Nível I & 6 & 12 & 11 & 14 & 13 & 6 \\
\hline & Nível II & 7 & 8 & 12 & 5 & 8 & 9 \\
\cline { 2 - 8 } & Nível III & 10 & 3 & 0 & 4 & 2 & 8 \\
\hline
\end{tabular}




\begin{tabular}{|l|l|l|l|l|l|l|l|}
\hline G2* & Nível I & 15 & 18 & 21 & 20 & 18 & 15 \\
\cline { 2 - 8 } & Nível II & 5 & 5 & 2 & 2 & 4 & 6 \\
\hline Nível III & 3 & 0 & 0 & 1 & 1 & 2 \\
\hline
\end{tabular}

Nível I - Normal; Nível II - Bom; Nível 3 - Excelente

Nota-se, na tabela 3, que a quantidade de crianças que atingiram os níveis II e III chega a ser superior no G1 quando comparado ao G2. Pelo menos $69,5 \%$ das crianças do G1 atingiram os níveis II ou III (bom ou excelente) enquanto no G2 apenas $26,1 \%$ atingiram os respectivos níveis.

Percebe-se que crianças que praticam o programa de motricidade (G1) têm mais probabilidade de melhorar seu desenvolvimento cognitivo do que crianças não praticantes.

\section{DISCUSSÃO}

Ao fazer correlação do desenvolvimento motor e cognitivo entre os sexos, nota-se que não foram detectadas nenhuma diferença significativa entre os grupos $(p=0,541)$. $\mathrm{Na}$ literatura ainda existe muita inconsistência correlacionado ao desenvolvimento motor de criança de sexo diferente. Nesse sentido, Lopes et al. (2003) relata que a evolução dos meninos é superior, pois eles apresentam mais oportunidade de prática de atividade física relacionado a assuntos culturais, ocasiões vivenciadas na infância e os tipos de jogos e brincadeiras, que são diferentes entre os sexos, discordando dos achados na presente pesquisa.

Entretanto, outros estudos constataram que o desenvolvimento motor de crianças até os 11 anos é linear, não há dissemelhança entre os dois sexos, porém a partir desta idade é capaz de perceber um maior desenvolvimento dos meninos em relação as meninas da mesma idade, corroborando com os achados da presente pesquisa (ROSA NETO et al., 2007; PEREIRA et al., 2010; MIRANDA et al., 2011; NOBRE, 2012). 
Os resultados deste estudo evidenciaram uma interação significativa entre os dois grupos (G1 x G2) participante e não participante, respectivamente nos escores motores da EDM e na avaliação Piagetiana. O grupo que participa do programa de intervenção alcançou um saldo positivo e significativo em todo os escores motores. Conforme a Figura 1, a média da Idade Motora Geral (IMG=75,04) mostrou-se superior em relação ao G2 da mesma maneira comportou-se a variável da Cognição, considerando uma resposta efetiva da intervenção ambiental (experiencias motoras). Tal comportamento não aconteceu no grupo (G2) que não frequenta o projeto, sendo possível observar que o grupo apresenta um atraso no desenvolvimento motor com relação à idade cronológica, já que a sua $I M G=38,61$ indica uma média de valores abaixo a sua $\mathrm{IC}=52,43$, mostrando um atraso considerável, resultando numa Idade negativa.

Quanto a classificação do QMG, foram identificados todos os sujeitos do grupo G1 tem demonstram maiores padrões de desenvolvimento (tabela 2). Desta forma, compreende-se que a maior parte das crianças se encontra num quociente motor elevado para idade cronológica. Aqui é possível inferências relevantes no contexto da efetividade do programa pois além do favorecimento para o desenvolvimento dos mesmos o programa talvez desempenhe papel relevante no avanço da motricidade melhorando inclusive aspectos cognoscitivo.

É oportuno destacar que na análise do desempenho motor e cognitivo, as crianças participantes do programa, expressaram modificações positivas e significativas apósavaliação nos escores cognitivo e motor, comprovando a primeira hipótese da pesquisa. Isso vem ao encontro de pesquisas anteriores que investigaram os benefícios da intervenção nos atrasos do desenvolvimento motor e da coordenação motora (WILLRICH; AZEVEDO; FERNANDES, 2008; MULLER, 2008; SILVA et al., 2011; NOBRE, 2012).

Para Caetano; Silveira e Gobbi (2005) tanto as circunstâncias quanto o ambiente em que a criança está inserida, bem como as exigências das tarefas sugeridas induzem grandiosamente o surgimento de novas habilidades. Em um estudo de revisão sistemática, o efeito da intervenção motora na evolução da criança nos primeiros anos 
de vida é sugerido como benéfica. Os autores destacam a relevância do tipo de intervenção direcionada pra cada intervalo de idade (BRAGA, 2009).

Observa-se nos resultados que a intervenção gera um estímulo positivo nas mudanças do desenvolvimento motor de cada criança que foram submetidas ao plano de treinamento elaborado para cada item psicomotor, pôde-se verificar que cada criança conseguiu adequar-se ao equiparado ou superior para sua faixa etária. No estudo de Nobre et al. (2012) evidenciou-se que maior parte das crianças submetidas as práticas desenvolvidas dentro de um programa de intervenção motora apresentaram melhor performance em práticas locomotoras e de controle de objeto em relação às crianças que não foram submetidas a um programa de intervenção.

Braga et al. (2009) analisaram pesquisas tanto nacionais quanto internacionais, associadas a intervenção, sendo que os resultados encontrados evidenciaram que após o período de estimulação do programa as crianças apresentaram evolução expressiva no desempenho motor.

Em estudo desenvolvido por Muller (2008) observou-se diferenças estatisticamente expressiva no programa motor concretizado, fortalecendo com isso a importância do desenvolvimento de atuações motoras para crianças com atraso motor e evidenciando que quanto mais cedo for diagnosticado este atraso, mais precoce se implementam propostas intervencionistas à fim de estimular o desenvolvimento motor da criança.

Em outro estudo, foi verificado o efeito de um programa de intervenção motora nos primeiros anos de vida de uma criança. Esses efeitos comprovaram que a atuação na fase inicial pode auxiliar nos ganhos do desenvolvimento humano e prevenir as incapacidades ou circunstâncias indesejáveis em indivíduos com atrasos no desenvolvimento (WILLRICH; AZEVEDO; FERNANDES, 2008).

Para Gallahue e Ozmun (2005) a criança dos 2 aos 9 anos de idade, está na fase de aquisição de movimentos fundamentais, consequentemente formaram uma base de toda uma aquisição motora seguinte. Vale-se pontuar que diversos autores justificam a relevância do desempenho proficiente em competências motoras fundamentais para 
processo de evolução das crianças (VALENTINI, 2007; GABBARD, 2008; STODDEN et al., 2008; HAYWOOD; GETCHELL, 2009; GOODWAY; ROBINSON; CROWE, 2010).

No presente estudo foi percebido mudanças confiáveis quanto as áreas psicomotoras, e cognitivas, o que revela que a criança inserida nesse contexto apresenta "evolução" nas áreas estimuladas dentro do programa de intervenção de motricidade. Esse fato evolutivo pode ser observado também na pesquisa feita por Campos et al. (2008), no qual verificou-se o efeito de um programa psicomotor voltado para crianças com baixo nível social. O resultado alcançado na pesquisa obteve um crescimento estatístico significativo na pontuação dos domínios de tonicidade, equilíbrio, praxia global, fina e estrutura espaço-temporal nas amostras analisadas, o que comprova a relevância de programas de interação para funcionamento psicomotor.

Os resultados evidenciados por esta pesquisa, possibilitam o reconhecimento da atuação do profissional de Educação Física enquanto um dos profissionais que integram uma equipe multiprofissional direcionada à intervenção precoce junto a criança com atraso no aperfeiçoamento da coordenação motora fina, global, equilíbrio e dentre outras variáveis. Dessa forma, o profissional de Educação Física especialista em psicomotricidade dispõe de funções específicas, como as realizadas neste estudo, como por exemplo, formação em psicomotricidade, a aplicação de instrumentos de avaliação específicos e a elaboração de um programa de estimulação que atenda as reais necessidades da criança em processo de desenvolvimento.

\section{CONCLUSÃO}

Os fundamentos abordados nesse estudo evidenciam o programa de motricidade e a importância da estimulação motora para o desenvolvimento global de crianças, podendo ser iniciado nos primeiros anos de vida. E através do seu corpo a criança conhece e descobre o mundo e experimenta sensações e as demonstram. Nessa coerência, torna-se relevante e necessário que todas as condições favoráveis estejam satisfatórias para que a criança possa integrar adequadamente as percepções e 
resposta aos estímulos, promovendo a união sensorial e a competência de exercer interação com o meio que a cerca.

Desta forma, considera-se que os componentes de um programa de motricidade quando estimulados precocemente proporcionam um desenvolvimento benéfico, evitando e mitigando limitações ao desenvolvimento, potencializando melhorias no processo motor e cognitivo. Portanto, crianças que praticam programa de motricidade têm mais probabilidade de melhorar seu desenvolvimento cognitivo do que crianças não praticantes.

\section{REFERENCIAS}

BRAGA, R. K. et al. A influência de um programa de intervenção motora no desempenho de habilidades loco motoras de crianças com idade entre 6 e 7 anos. Revista de Educação Física/UEM, Maringá, 20 ( 2): 171181, 2009.

CAETANO, M. J. D.; SILVEIRA, C. R. A.; GOBBI, L. T. B. Desenvolvimento motor de pré-escolares no intervalo de 13 meses. Rev. Bras. Cineantropom. Desempenho Hum, 7 (2): 05-13, 2005.

CAMPOS, A. C; SILVA, L. H.; PEREIRA, K. ROCHA, N. A. C; TUDELLA, E. Intervenção psicomotora em crianças de nível socioeconômico baixo. Fisioter. Pesqui. 15(2): 188-93, 2008

LOPES, V.P. et al. Estudo do nível de desenvolvimento da coordenação motora da população escolar (6 a 10 anos de idade) da Região Autónoma dos Açores. Revista Portuguesa de Ciências do Desporto, 3 (1): 47-60, 2003.

GALLAHUE, D. L. \& OZMUN, J. C. Compreendendo o desenvolvimento motor: bebês, crianças, adolescentes e adultos. 3.ed. São Paulo: Phorte Editora. 2005.

GABBARD, C. Lifelong Motor Development. 5 ed. Texas: Benjamin Cumming, 2008. 
GOODWAY, J. D.; ROBINSON, L. E.; CROWE, H. Gender differences in fundamental motor skill development in disadvantaged preschoolers from two geographical regions. Research quarterly for exercise and sport, 81(1):17-24, 2010.

HAYWOOD, K. M.; GETCHELL, N. Desenvolvimento motor ao longo da vida. Porto Alegre: Artmed. 2009.

HULley, S. B.; NeWMAN, T. B.; CUMMINGS, S. R. Primeira Parte: Anatomia e Fisiologia da Pesquisa Clínica. In: Hulley, Stephen B.; Cummings, Steven R.; Browner, Warren S. et al. Delineando a pesquisa clínica: uma abordagem epidemiológica. $2^{\underline{a}}$ Ed. Porto Alegre: Artmed, p: 21-34, 2003.

KING-THOMAS L. A therapist's guide to Pediatric Assessment. Boston: Little Brown, 1987.

MIRANDA, T. B.; BELTRAME, T. S.; CARDOSO, F.L. Desempenho motor e estado nutricional de escolares com e sem transtorno do desenvolvimento da coordenação. Rev. bras. cineantropom. desempenho hum, 13 (1):59-66, 2011.

MOURA, M. C. Organização do espaço: contribuições para uma educação de qualidade. 2009. Dissertação (Mestrado em Educação) Universidade de Brasília. Brasília, 2009.

MULLER, A. B. Efeitos da Intervenção motora em diferentes contextos no desenvolvimento da criança com atraso motor. 2008. 186 f. Dissertação (Mestrado em Movi- mento Humano) - Universidade Federal do Rio Grande do Sul, Rio Grande do Sul, 2008.

NOBRE, F. S. S. et al. Intervenção motora como fator determinante no desenvolvimento motor: estudo com- parativo e quase experimental. Revista Acta Brasileira do Movimento Humano, Santa Catarina, 2 (2): 76-85, 2012. 
Pereira, P. Manzatto, L. Marco, A. Análise do crescimento e desenvolvimento moto de escolares de $1^{\text {a }}$ a $4^{a}$ série do município de Holambra - São Paulo. HU Revista, Juiz de Fora, v.36, n. 4, p. 308-314, out/dez 2010.

PIAGET, J. Seis estudos de psicologia. São Paulo: Editora Forense, 1998.

Rosa Neto, F. Manual de avaliação motora. 2a ed. Porto Alegre: Artmed; 2014.

ROSA NETO. F. et al. Desenvolvimento Motor de crianças com indicadores de dificuldades na aprendizagem escolar. Rev. bras. Ci e Mov, 15 (1): 45-51, 2007.

SANTOS, S.; DANTAS, L. T.; OLIVEIRA, J. A. Desenvolvimento motor de criancas, de idosos e de pessoas com transtornos da coordenação. Revista Paulista de Educação Física, São Paulo, 18 (esp):33-44, 2004.

SANTOS, C. et al. Proposta de protocolo de exercícios, baseado na relação do equilíbrio e da coordenação motora com os hábitos de vida diária de crianças de sete anos. Revista Brasileira de Ciências da Saúde, São Caetano do Sul, ano 3 (11): 8-15, 2007.

SILVA, E. V. A. et al. Programa de intervenção motora para escolares com indicativo de transtorno do desenvol- vimento da coordenação. Revista Brasileira de Educação Especial, Marília, 17 (1): 137-150, 2011.

SILVA, C. R. O. Metodologia e organização do projeto de pesquisa: guia prático. Fortaleza, CE: Editora da UFC, 2004.

STODDEN, D.F. et al. Developmental perspective on the role of motor skill competence in physical activity: an emergent relationship. Quest, 60 (1):290-306, 2008.

WILLRICH, A.; AZEVEDO, C. C. F.; FERNANDES, J. O. Desenvolvimento motor na infância: influência dos fatores de risco e programas de intervenção. Revista de Neurociencias, São Paulo, 17 (1): 51-56, 2008 
VALENTINI, N. C. Competência Percebida: Considerações para promover a aprendizagem. Em: Krebs RJ, Ferreira Neto CA. Tópicos em desenvolvimento motor na infância e adolescência. Rio de Janeiro: Editora LECSU, p. 137-157, 2007.

Enviado: Julho, 2020.

Aprovado: Agosto, 2020. 